\title{
Niche Required for Inducing Quiescent Stem Cells
}

\author{
S.-I. Nishikawa, M. Osawa, S. Yonetani, S. Torikai-Nishikawa, And R. Freter \\ Laboratory for Stem Cell Research, RIKEN Center for Developmental Biology, Kobe, Japan
}

\begin{abstract}
Quiescence is an important feature distinguishing stem cells (SCs) from other compartments for most SC systems. Evidence suggests that the quiescent state is directed by external cues expressed in the presumptive microenvironment, the niche, although the cellular and molecular nature of the niche remains obscure in most SC systems. Our group has been addressing this question using the melanocyte (MC) as a model, because MC SCs (MSCs) and other compartments are distinguished by their location in the hair follicle, the former in the bulge and the other in the hair matrix. On the basis of the gene expression profiles of MSCs, we developed a method to distinguish MSCs from other compartments by using their own characteristics. Using the new criterion for MSCs, we investigated the molecular cues that induce the quiescent MSCs from proliferating melanoblasts. Our study showed that fibroblast growth factor-2 (FGF-2), or an equivalent signal, is essential for inducing a set of MSC signatures, although additional signals required for inducing the ultimate MSCs remain to be identified.
\end{abstract}

Niche is an important issue common to all types of SCs (Spradling et al. 2001; Ohlstein et al. 2004; Moore and Lemischka 2006; Scadden 2006). To the best of our knowledge, however, in no mammalian SC system have cells responsible for niche function ever been specified in such an exact manner as has been shown in the gonads of Drosophila and Caenorhabditis elegans. Indeed, the niche cells for germ cells of these species (such as tip cell and cap cell) have been fully defined (Spradling et al. 2001; Ohlstein et al. 2004). In contrast, the SCs in mammalian tissues have been characterized to a considerable extent by their own features, and they are now able to be specified in a relatively precise manner (Fuchs et al. 2004; Joseph et al. 2004). Together, SC research in mammals remains in a situation where the niche can be defined no more than the presumptive area in the vicinity of SCs.

Although the situation for the MSC system is not different from that of other SC systems, there are appreciable advantages in MCs for studying the niche. In this chapter, we summarize our recent studies on MSCs in the hair follicle, particularly the quiescent SCs, and present our views on the microenvironment required for inducing the quiescent SCs.

\section{MSCS SERVE AS AN IDEAL MODEL TO UNDERSTAND QUIESCENCE OF SCS}

Two types of SC systems exist in the vertebrate body: a constitutive type, such as gut epithelium in which SCs undergo continuous self-renewal, and the other, a regenerative system in which SCs repeat the quiescence and regeneration cycles with a variable length of the quiescent stage. The MSC system is a typical example of the regenerativetype system, and its regeneration cycle is linked to that of follicular keratinocytes (hair cycle). MSCs are functionally distinguished from other compartments of the MSC system by a number of features (Nishimura et al. 2002). First, in mature hair follicles, MSCs are distinguished by their



Figure 1. Architecture of the mouse hair follicle. (Left) A hair follicle is divided into permanent and transient portions. Only a transient portion undergoes apoptosis/regeneration cycles. Previous studies demonstrated that SCs for follicular keratinocytes are present in the bulge region. Most MCs in hair follicles are present in the hair matrix at the bottom of follicles. MCs are also found in the most lower portion of the permanent region, which corresponds to MSCs. (Right) Whole-mount staining of the lower permanent portion (LPP) of a hair follicle. MSCs are visualized by anti-LacZ staining. MSCs exist solitarily.

location in the hair follicles. As shown in Figure 1, a hair follicle is divided into two parts: the upper permanent portion and the lower transient portion. Only the transient portion is renewed in each hair cycle. The hair cycle is mainly directed by the hair papilla that localizes at the bottom of 
the follicles and emanates a set of molecules regulating the activities of follicular keratinocytes and the MC. Hence, all proliferating and differentiating MCs need to localize in the hair matix in the vicinity of the hair papilla. In mouse skin, MCs cannot survive outside the hair matrix. However, the small region at the bottom of the permanent portion that includes a region called the bulge is the exception, because this region has a distinctive capacity to serve as the niche capable of supporting the survival of the quiescent MSCs. In this chapter, we designate this region as the lower permanent portion (LPP). This invariable definition of MSCs in terms of its location allows further characterization of MSCs. In fact, we have shown that MSCs in the LPP correspond to quiescent SCs, because they do not proliferate until a new hair regeneration cycle is initiated and they become resistant to deprivation of the c-Kit signal.

Another unique feature of MSCs, as compared with other $\mathrm{SC}$ systems, is that they can exist solitarily separated from other components of the MSC system. The LPP usually contains multiple MSCs, but it is possible, under certain experimental conditions, to prepare hair follicles that contain only a single MSC in the LPP. Moreover, Figure 1 shows that, even in the LPP containing multiple MSCs, each MSC exists solitarily without notable connections to one another (Nishimura et al. 2002; Mak et al. 2006). Hence, if there exists a distinctive niche for MSC, it should comprise heterologous cells, most likely keratinocytes. On the other hand, in many SC systems including the hair follicle, SCs are integrated in a continuous cluster together with other compartments of the SC systems. For instance, SCs of the gut epithelium are wedged by their immediate progeny (Potten et al. 2003). In such a situation, it is difficult to rule out the possibility that the progeny of SCs themselves are involved in the function of the niche, thereby making the cellular composition of the niche more complicated. Indeed, Hans Clevers (this volume) demonstrated that gut epithelial cells autonomously generate an SC system in the absence of heterologous cell lineages.

In conclusion, an explicit definition of SCs and the simplicity of the cellular components of the niche point to the MSC as an ideal model for studying the SC niche.

\section{CELLULAR COMPARTMENTS IN THE MSC SYSTEM}

All skin MCs are derived from neural crest cells that spread over the whole body surface through the subcutaneous mesenchyme, enter the developing epidermis, and eventually settle in hair follicles. In mouse skin, because of the absence of stem cell factor (SCF), MCs cannot survive in the dermis or interfollicular epidermis. Hence, mouse MCs are present exclusively in the hair follicle, except for regions such as the auricle and palm where MCs are found in dermis. Interestingly, when the scf transgene is expressed in the epidermis, MCs also become distributed in the interfollicular epidermis (Kunisada et al. 1998). As a result, localization of MCs is directed extrinsically, rather than according to the cell-autonomous program. This passivity is another unique characteristic of the MSC system. Figure 2 summarizes our current understanding of the fate divergence of the embryonic melanoblast (MB) in postna-



Embryonic MB Neonatal MB Differentiation

Figure 2. Divergence of the MB in neonatal skin. During embryogenesis, MBs expand and migrate to hair follicles. This process is regulated by various molecules including SCF and endothelin expressed in embryonic dermal tissues. After birth, the MB undergoes fate divergence. Some that migrate to the hair matrix undergo irreversible commitment to differentiated MCs under the control of molecules emanated from hair papilla. The rest migrate to the LPP and differentiate to MSCs. MSCs are reactivated by yet unknown mechanisms. Some of the reactivated MBs can maintain the capacity to revert to MSCs, when they meet the niche in the LPP. However, most of reactivated MBs migrate to the hair matrix and give rise to differentiated MCs.

tal life. During embryogenesis, the MB maintains selfrenewal with only a limited rate of differentiation. After entering developing hair follicles, those migrating into the hair matrix undergo terminal differentiation, whereas those trapped in the LPP are induced to become quiescent MSCs that can be reactivated in the next hair cycle (Nishimura et al. 2002). Of note is that some of progeny of the reactivated MSCs can maintain the ability to revert to MSCs. Nonetheless, all of the states are regulated by extrinsic cues present in distinct environments.

\section{MSC SIGNATURE}

As described above, LPP localization is the most reliable mark for MSC signature. Thus, we first isolated individual MSCs by manual dissection of hair follicles. Using transgenic mice that were engineered to express green fluorescent protein (GFP) specifically in the MC lineage, we could distinguish MSCs from other cells in the LPP. We next prepared a single-cell cDNA library of harvested MSCs, analyzed gene expression profiles, and compared them with those prepared from MBs in the embryonic epidermis and MCs in the hair matrix. Figure 3 presents characteristic gene expression profiles in these three MC populations. Despite unavoidable variation due to technical difficulties in analyzing gene expression at the single-cell level, MCs collected from the LPP are significantly similar in gene expression (Osawa et al. 2005). Moreover, the overall gene expression profiles of the three populations are different from one another, although some variations are observed among individual cells in the same group.

Taken together, this single-cell analysis defines two molecular features of MSCs: a low level of housekeeping gene expression and down-regulation of MC-specific genes. 

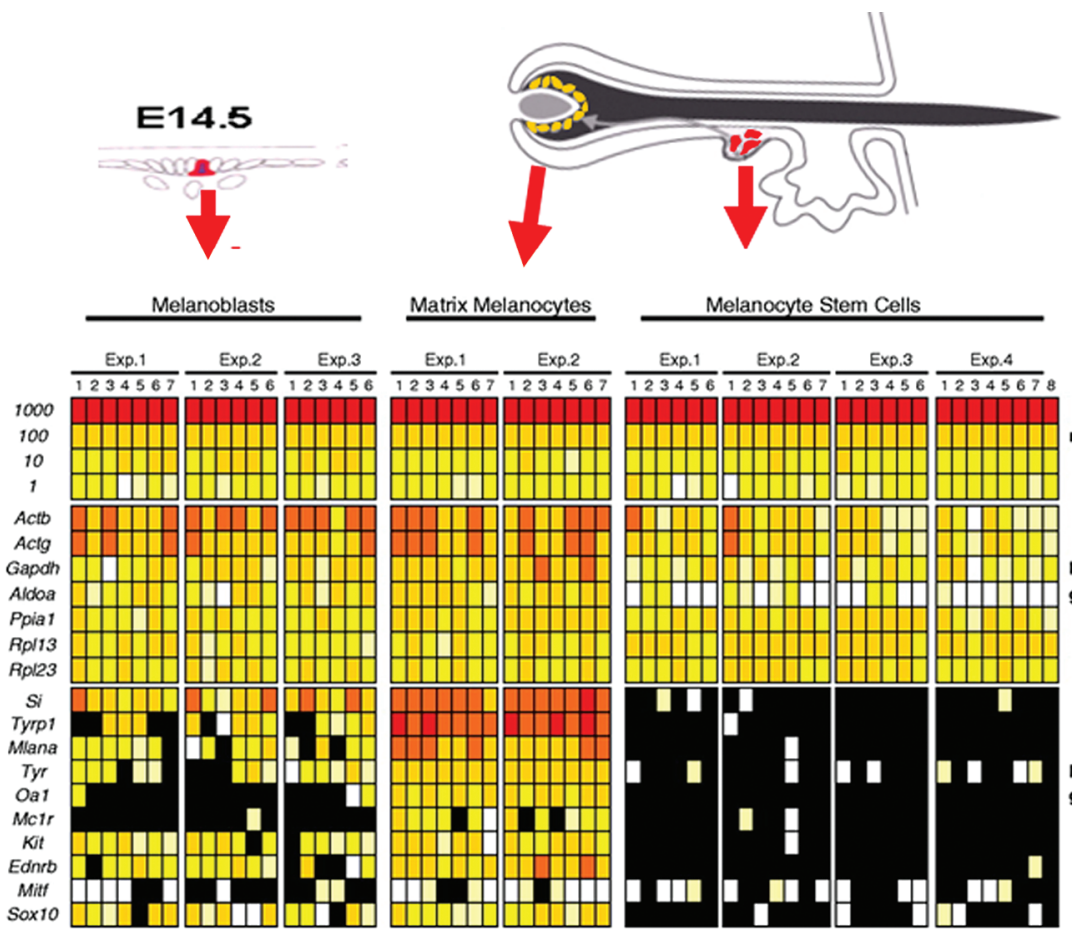

mRNA spikes

Housekeeping

genes

Fire 3. Gene expression profiles of MCs in embryos, hair matrix, and LPP. (Top) Individual MCs are isolated from embryonic skin, hair matrix, and LPP. RNA was prepared from each individual cell and amplified. Expression levels of genes in single-cell cDNA libraries were analyzed by quantitative polymerase chain reaction. Expression levels were classified into five grades and expressed as black, white, yellow, light orange, and orange. Each column represents the data of each cell. (Modified from Osawa et al. 2005.)

\section{USE OF FACS FOR ANALYZING MSC FEATURES}

The aforementioned data on gene expression of each individual MSC suggest additional methods to specify MSCs. The first method is to use the low expression level of MSC housekeeping genes. Because the method that we used for distinguishing MCs from other lineages is the chicken $\gamma$-actin (CAG) promoter-driven $g f p$ gene, we expected that the expression level of the housekeeping genes could be monitored by measuring GFP expression level. As expected, the expression level of $g f p$ in MC lineage cells in hair follicles from P10 skin varies to a considerable extent (Fig. 4). To verify whether MSC is included in the GFPlow population, we took advantage of another feature of MSCs in neonatal skin: the resistance against starvation of SCF. Previously, we showed that treatment of neonatal mice by ACK2, an antagonistic monoclonal antibody to c-Kit, depletes nearly all MCs from the skin except for those that colonize to the LPP. Thus, MCs surviving after ACK2 treatment are likely to contain MSCs and are expected to be GFPlow. In fact, ACK2 treatment depleted all of the GFPhigh population, whereas the GFPlow population survived. This GFP ${ }^{\text {low }}$ population is easily purified by fluorescence-activated cell sorting (FACS), and we were able to confirm that the GFPlow cells share the many characteristcs of MSCs such as down-regulation of a group of MC-specific genes such as trp2 and sox10.

The other method for distinguishing MSCs by FACS is to use the $g f p$ gene driven by promoters of MC-specific
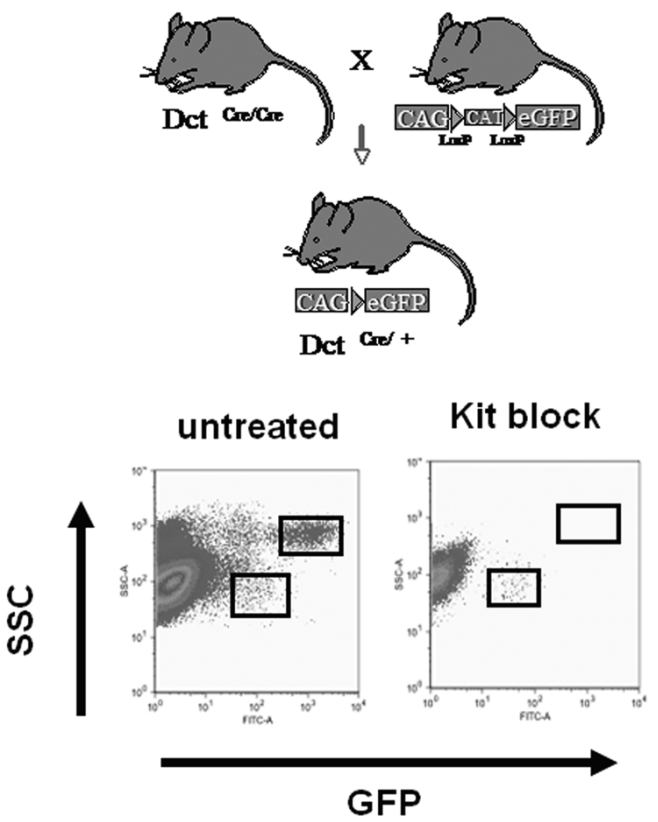

Figure 4. Low activity of the chicken $\gamma$-actin (CAG) promoter in MSCs. (Top) Generation of mice that express GFP driven by the CAG promoter in MCs. The Dct-Cre mouse was a kind gift from Dr. F. Beermann (Guyonneau et al. 2002). Mice were mated with a transgenic strain that bears the CAG-driven GFP gene activated by Cre recombinase. (Bottom) FACS analysis of cells dissociated from neonatal skin. (Right panel) Analysis of untreated mice. Two $\mathrm{GFP}^{+}$populations are found. (Left panel) Analysis of mice injected with anti-c-Kit monoclonal antibodies (ACK2) at birth. Only GFPlow cells remain in the skin preparation. 
genes. We have been trying to confirm this possibility by generating two mouse strains harboring the $g f p$ gene, one driven by the $d c t$ promoter and the other driven by the sox 10 promoter. Up to now, we have completed analysis of the dct-gfp transgenic mouse and confirmed that the MC of P10 mouse skin contains both GFPhigh and GFPlow populations. Analysis of sox 10-gfp mice is currently in progress.

These results clearly indicate that the two features of MSCs defined originally by analysis at the single-cell level can now be used for defining MSCs at the population level by using FACS. This progress is essential for distinguishing MSCs from other compartments without using histological localization.

\section{GENETIC APPROACH TO INVESTIGATE THE MOLECULAR MECHANISMS UNDERLYING MSC MAINTENANCE}

This progress also allows us to use FACS to sort the MSC population en mass. Comparing CAG-driven GFP high and low populations, we were able to list genes that are expressed at higher levels in MSCs. Of importance is that despite low expression of some housekeeping genes and MC-specific genes, we found genes that were expressed at higher levels in MSCs. Molecules involved in Notch signaling are an example that were listed to be expressed at higher levels. An advantage of MC lineage is that complete absence of MCs does not have much of an effect on the life of the animal. This unique feature of MCs allows us to evaluate the role of a particular molecule on MC activity during a long period of time. As is the case for some of the other cell lineages, a number of MC-specific gene expression systems have been developed that are used for MC-specific gene knockouts. Using these advantages, we have investigated the function of the Notch signal in the development and maintenance of the MSC system (Moriyama et al. 2006). Our results unequivocally demonstrate the role of Notch in the development and maintenance of MSCs.

\section{INDUCTION OF QUIESCENT MSCS}

In the mouse, the $\mathrm{MB}$ undergoes the final differentiation to MSCs as late as about P4, because this is the stage when the functional niche is formed in the LPP. Despite this, the cellular identity of niche has not yet been clarified; the timing of the niche formation was able to be estimated through the analysis of the $b c l 2^{--}$mouse. In the $b c l 2^{--}$mouse, the MB undergoes faster apoptosis upon SCF starvation. Thus, neonatal ACK2 injection resulted in the complete absence of MSCs in hair follicles due to rapid depletion of MBs before the formation of the niche (Mak et al. 2006). On the other hand, in the wild-type mouse, a portion of the MB can survive for more than 3 days against SCF starvation and is able to meet the niche that is newly formed in the LPP of guard hairs. Nonetheless, all key events required for induction of MSCs should occur during these neonatal 4 days.

To dissect this process, we analyzed the timing of the appearance of distinct MSC features. Our histological analysis showed that Sox 10 expression in MCs is downregulated at about $\mathrm{P} 2$ in the upper area of the developing hair follicle. FACS analysis showed that cells that are low in $d c t$-promoter-driven $g f p$ are first detected at E18.5. These results suggests that, even among MC-specific genes that are down-regulated in MSCs, the timing of when each individual feature appears is variable. Likewise, cells that show low expression of CAG-promoter-driven GFP were first detected at E18.5. Concerning the resistance to ACK2 treatment (resistance against SCF starvation), which we are using to specify MSCs in neonatal hair follicles, our previous studies showed that such a state is also observed in embryonic stages. Indeed, MBs in E15.5-17.5 embryos are ACK2-resistant (Yoshida et al. 1993). As such, it is likely that each feature associated with the MSCs in the LPP is acquired through multiple and probably independent steps, rather than a single step. If so, it is important to define each distinct MSC signature in terms of function significance. In this respect, it must be emphasized that the functional definition of the ultimate SCs has not yet been fully attained.

\section{ATTEMPT TO INDUCE QUIESCENT MSCS IN VITRO}

As described above, genetic methods are available for manipulating genes in the MC lineage. Thus, determining the functional role of each individual molecule in MSC activity may be feasible. Moreover, there is a definite conception about the phenotype resulting from the defect in this process. Because MSC differentiation was completed later than the colonization of MBs to the hair matrix, defects specifically affecting the MSC should be expressed such that mice born pigmented lose pigmentation in the next hair cycle. Thus, a continuous effort to generate conditional knockout strains of MSC signature genes is important for understanding molecular mechanisms involved in induction and maintenance of MSCs.

What might be difficult with this genetic approach, although not impossible, is to determine a minimum requirement for a given process, e.g., the molecular requirement for inducing MSCs. As shown in the preceding sections, the induction of MSCs is likely to be regulated by multiple extrinsic signals. We think that in vitro culture of embryonic MCs may address this issue more effectively, because in vitro culture per se is a constitutive approach to recapitulate the process. With this in mind, we decided to use MB culture to search for the minimum requirements for inducing quiescent MSCs.

Although we have established a method for distinguishing MSCs from other compartments by FACS, culturing FACS-purified MBs is a new challenge that has not yet been reported. In fact, all previous culture conditions for MBs were optimized for MBs mixed with other skin cells. We have tested those culture conditions reported previously (Hirobe 1991; Sviderskaya et al. 1995), but none of them were able to support the proliferation of FACS-purified MBs. On the other hand, culture with the XB2 (Rheinwald and Green 1975) feeder layer originally developed by Bennett's group (Sviderskaya et al. 1995) turned out to be capable of supporting clonogenic proliferation of FACS-purified MCs (Yonetani et al. 2008). The proliferation of MCs under these conditions is c-Kit-dependent, and 
its differentiation is promoted by endothelin. More importantly, MBs proliferating in this culture are able to reconstitute the whole MSC system in hair follicles regenerated from dissociated keratinocytes. This result shows unequivocally that at least a portion of the cells in this culture can replenish MSCs in vivo. This culture can now be used to explore extrinsic signals that induce various features of MSCs and eventually the quiescent MSCs.

Using this culture system, we have been searching for molecules that are able to induce SC signature. XB2+SCF supports sustained proliferation of $\mathrm{MBs}$, but the MSC signature was not induced. Among the molecules that we have tested in this culture, basic FGF showed a striking activity to induce CAG-GFPlow as well as Dct-GFP low populations. Those two populations are indeed overlapped (S. TorikaiNishikawa et al., unpubl.). We are currently examining whether other features of MSCs such as low-Sox 10 activity are associated with this state, but our preliminary analysis suggests that the Sox 10 expression level in the CAG-GFPlow population is comparable to that of GFPhigh $\mathrm{MBs}$, even though they are in GFPlow populations. Hence, the final state of the quiescent MSCs is not attained by basic FGF alone. On the other hand, cells that are slowly cycling and ACK2resistant are indeed induced in this FGF-induced GFPlow population. Further attempts to define molecules required for inducing all MSC features are currently in progress.

\section{CONCLUSION}

The MSC system has a unique advantage for studying the niche for the quiescent MSCs. Our study suggests, however, that the quiescent MSCs are induced not by a single encounter with the niche, but rather through multiple steps acquiring each distinct MSC feature. After extensive analyses of in vivo MSCs, we are now able to investigate the signals involved in this process by using in vitro culture. Although the molecular mechanisms inducing and maintaining the ultimate MSCs remain totally obscure, some signals such as basic FGF that are involved in inducing quiescence, as well as some features of MSCs, are being uncovered.

\section{ACKNOWLEDGMENTS}

This study is supported by the Leading Project for Realization of Regenerative Medicine.

\section{REFERENCES}

Fuchs, E., Tumbar, T., and Guasch, G. 2004. Socializing with the neighbors: Stem cells and their niche. Cell 116: 769-778.

Guyonneau, L., Rossier, A., Richard, C., Hummler, E., and
Beermann, F. 2002. Expression of Cre recombinase in pigment cells. Pigment Cell Res. 15: 305-309.

Hirobe, T. 1991. Selective growth and serial passage of mouse melanocytes from neonatal epidermis in a medium supplemented with bovine pituitary extract. J. Exp. Zool. 257: 184 194.

Joseph, N.M., Mukouyama, Y.S., Mosher, J.T., Jaegle, M., Crone, S.A., Dormand, E.L., Lee, K.F., Meijer, D., Anderson, D.J., and Morrison, S.J. 2004. Neural crest stem cells undergo multilineage differentiation in developing peripheral nerves to generate endoneurial fibroblasts in addition to Schwann cells. Development 131: 5599-5612.

Kunisada, T., Yoshida, H., Yamazaki, H., Miyamoto, A., Hemmi, H., Nishimura, E., Shultz, L.D., Nishikawa, S., and Hayashi, S. 1998. Transgene expression of steel factor in the basal layer of epidermis promotes survival, proliferation, differentiation and migration of melanocyte precursors. Development 125: 2915-2923.

Mak, S.S., Moriyama, M., Nishioka, E., Osawa, M., and Nishikawa, S. 2006. Indispensable role of $\mathrm{Bcl} 2$ in the development of the melanocyte stem cell. Dev. Biol. 291: 144-153.

Moore, K.A. and Lemischka, I.R. 2006. Stem cells and their niches. Science 311: 1880-1885.

Moriyama, M., Osawa, M., Mak, S.S., Ohtsuka, T., Yamamoto, N., Han, H., Delmas, V., Kageyama, R., Beermann, F., Larue, L., and Nishikawa, S. 2006. Notch signaling via Hes1 transcription factor maintains survival of melanoblasts and melanocyte stem cells. J. Cell Biol. 173: 333-339.

Nishimura, E.K., Jordan, S.A., Oshima, H., Yoshida, H., Osawa, M., Moriyama, M., Jackson, I.J., Barrandon, Y., Miyachi, Y., and Nishikawa, S. 2002. Dominant role of the niche in melanocyte stem-cell fate determination. Nature 416: 854 860.

Ohlstein, B., Kai, T., Decotto, E., and Spradling, A. 2004. The stem cell niche: Theme and variations. Curr. Opin. Cell Biol. 16: 693-699.

Osawa, M., Egawa, G., Mak, S.S., Moriyama, M., Freter, R., Yonetani, S., Beermann, F., and Nishikawa, S. 2005. Molecular characterization of melanocyte stem cells in their niche. Development 132: 5589-5599.

Potten, C.S., Booth, C., Tudor, G.L., Booth, D., Brady, G., Hurley, P., Ashton, G., Clarke, R., Sakakibara, S., and Okano, H. 2003. Identification of a putative intestinal stem cell and early lineage marker; musashi-1. Differentiation 71: 28-41.

Rheinwald, J.G. and Green, H. 1975. Formation of a keratinizing epithelium in culture by a cloned cell line derived from a teratoma. Cell 6: 317-330.

Scadden, D.T. 2006. The stem-cell niche as an entity of action. Nature 441: 1075-1079.

Spradling, A., Drummond-Barbosa, D., and Kai, T. 2001. Stem cells find their niche. Nature 414: 98-104.

Sviderskaya, E.V., Wakeling, W.F., and Bennett, D.C. 1995. A cloned, immortal line of murine melanoblasts inducible to differentiate to melanocytes. Development 121: 1547-1557.

Yonetani, S., Moriyama, M., Nishigori, C., Osawa, M., and Nishikawa, S. 2008. In vitro expansion of immature melanoblasts and their ability to repopulate melanocyte stem cells in the hair follicle. J. Invest. Dermatol. 128: 408-420.

Yoshida, H., Nishikawa, S., Okamura, H., Sakakura, T., and Kusakabe, M. 1993. The role of c-kit proto-oncogene during melanocyte development in mouse. In vivo approach by the in utero microinjection of anti-c-kit antibody. Dev. Growth Differ. 35: 209-220. 


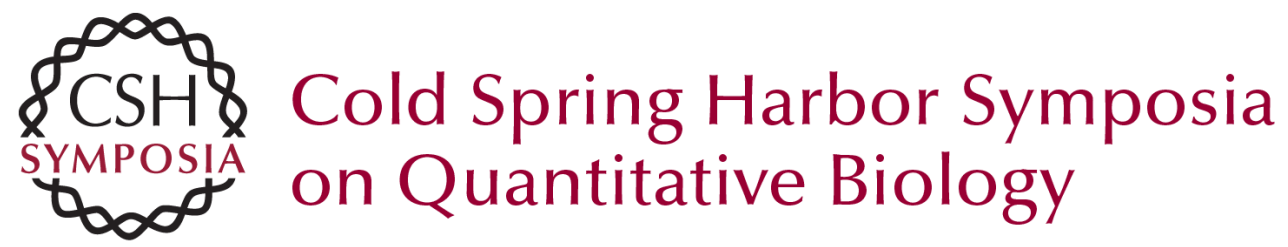

\section{Niche Required for Inducing Quiescent Stem Cells}

S.-I. Nishikawa, M. Osawa, S. Yonetani, et al.

Cold Spring Harb Symp Quant Biol 2008 73: 67-71 originally published online November 6, 2008 Access the most recent version at doi:10.1101/sqb.2008.73.024

References This article cites 18 articles, 6 of which can be accessed free at: http://symposium.cshlp.org/content/73/67.full.html\#ref-list-1

License

Email Alerting Receive free email alerts when new articles cite this article - sign up in the box at the Service top right corner of the article or click here.

To subscribe to Cold Spring Harbor Symposia on Quantitative Biology go to: http://symposium.cshlp.org/subscriptions 